\title{
Time-domain electric field enhancement on micrometer scale in coupled split ring
} resonator upon terahertz radiation

\author{
Lange, Simon Lehnskov; Iwaszczuk, Krzysztof; Hoffmann, Matthias; Broeng, Jes; Jepsen, Peter Uhd
}

Published in:

Proceedings of 2016 41st International Conference on Infrared, Millimeter, and Terahertz waves

Link to article, DOI:

10.1109/IRMMW-THz.2016.7758702

Publication date:

2016

Document Version

Peer reviewed version

Link back to DTU Orbit

Citation (APA):

Lange, S. L., Iwaszczuk, K., Hoffmann, M., Broeng, J., \& Jepsen, P. U. (2016). Time-domain electric field enhancement on micrometer scale in coupled split ring resonator upon terahertz radiation. In Proceedings of 201641 st International Conference on Infrared, Millimeter, and Terahertz waves (pp. 1-2). IEEE Press. International Conference on Infrared, Millimeter and Terahertz Waves https://doi.org/10.1109/IRMMWTHz.2016.7758702

\section{General rights}

Copyright and moral rights for the publications made accessible in the public portal are retained by the authors and/or other copyright owners and it is a condition of accessing publications that users recognise and abide by the legal requirements associated with these rights.

- Users may download and print one copy of any publication from the public portal for the purpose of private study or research.

- You may not further distribute the material or use it for any profit-making activity or commercial gain

- You may freely distribute the URL identifying the publication in the public portal 


\title{
Time-domain electric field enhancement on micrometer scale in coupled split ring resonator upon terahertz radiation
}

\author{
Simon L. Lange ${ }^{1}$, Krzysztof Iwaszczuk ${ }^{1}$, Matthias Hoffmann ${ }^{2}$, Jes Broeng ${ }^{1}$ and Peter U. Jepsen ${ }^{1}$ \\ ${ }^{1}$ DTU Fotonik, Technical University of Denmark, DK-2800 Kgs. Lyngby, Denmark \\ ${ }^{2}$ SLAC National Accelerator Laboratory, Menlo Park, California, United States
}

\begin{abstract}
We present here a novel design for a coupled split ring resonator antenna optimized for time-domain electric field enhancement in the 0.1 to 1 terahertz (THz) range. The antenna is designed to be sensitive to the incident field polarization and seeks to avoid metal damage due to electron bombardment.
\end{abstract}

\section{INTRODUCTION}

$\mathrm{R}$ ECENT research has shown ultrafast electron field emission from metal antennas due to strong terahertz pulse irradiation $[1,2]$. The emission is strongly nonlinear with the electric field strength. The presented work investigates a design for a polarisation dependent, coupled split ring resonator antenna. The antenna enhances the peak electric field in a large, few $\mu \mathrm{m}$-sized gap designed to avoid metal damage due to electron bombardment as well as nonlinear effects in the antenna substrate [3]. The electron field emission is intended to excite surrounding gas molecules that subsequently undergo irradiative relaxation in the CCD sensitive UV regime. Placing the antenna in a periodic fashion will therefore constitute a direct measurement detector for $\mathrm{THz}$ radiation with a spatial, lateral resolution on the order of $200 \mu \mathrm{m}$ and a strong dependency on the incident polarisation.

Very little work has been reported on antennas optimized for field enhancement in the time domain. Most interestingly, a giant field enhancement of $10^{4}$ in the time domain has been shown by using a single split-ring resonator with a nanometersized gap [4]. Photoconductive, fractal antennas have been shown to work well at multiple $\mathrm{THz}$ frequencies, thus having a broadband emission spectrum. However, the emission comes from different points in space and time.

Based on all the above-mentioned findings, the design realized in this work has a list of features. A) it has a single gap that is small enough for plasmonic coupling between the tips. B) it utilizes the resonant nature of a dipole antenna to enhance specific frequencies in the incoming $\mathrm{THz}$ transient. C) it makes use of the fractal antenna idea where multiple currents can be brought to interfere constructively and create multiple resonance frequencies. D) it encapsulates the field in a way that could prevent coupling between antennas when put closely in a large array for $\mathrm{THz}$ detection.

It has been observed in [5] that ultrafast impact ionization and carrier generation occurs in high resistivity silicone (HR$\mathrm{Si}$ ) when illuminated with intense subpicosecond $\mathrm{THz}$ transients. Therefore, a HR-Si substrate is combined with a layer of $\mathrm{SiO}_{2}$ on top in this work. The former has a negligible loss in the $\mathrm{THz}$ region whereas the latter has a higher breakdown voltage and lower electron mobility, so induced nonlinearities due to the high field strength will be less pronounced in $\mathrm{SiO}_{2}$ than in $\mathrm{HR}-\mathrm{Si}$.

\section{RESUlts}

A HR-Si substrate is illuminated from the back with a $4 \mathrm{ps}$ short terahertz pulse, which is linearly polarized in the positive $y$-direction. On the top, $1 \mu \mathrm{m} \mathrm{SiO}_{2}$ is deposited and subsequently a $200 \mathrm{~nm}$ thick Au antenna structure - see Fig. 1. The two main current loops are designed such that the ratio between the circumferences is exactly 1.5. Finite-Element Time-Domain (FETD) simulations show a field enhancement in time domain of 350 . The simulations assume a linear material response to the incident field.

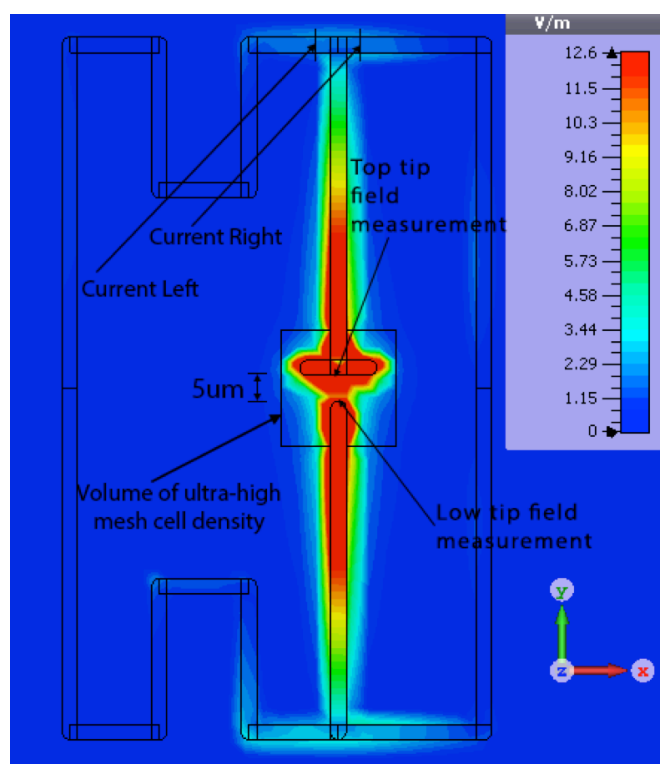

Fig. 1: Antenna structure seen from the top at the point in time when the field enhancement is the largest. The colour bar is saturated at about $5 \%$ of the maximum field to show the detailed distribution better. The incident polarisation is linear in the $y$-direction and the incident field strength is set to $1 \mathrm{~V} / \mathrm{m}$. Hence, the figure effectively shows the field enhancement.

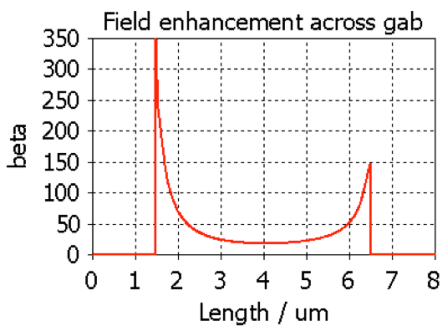

Fig. 2: The field enhancement across the gap going from the low tip to the top tip $100 \mathrm{~nm}$ above the substrate surface. The effect of the perpendicular bar on the top tip is a lower field enhancement than for the pointy, low tip. 
The role of the $\mathrm{SiO}_{2}$ is partly to push a significant portion of the field into free space compared to having a pure $\mathrm{Si}$ substrate. This push occurs as the field tends to concentrate in high refractive index regions and $\mathrm{n}_{\mathrm{SiO} 2}=1.95<\mathrm{n}_{\mathrm{Si}}=3.45$. The effect is apparent in Fig. 3, which is a cross-sectional view in the center of the gap depicted in Fig. 1. The black lines show the $\mathrm{SiO}_{2}$ layer.

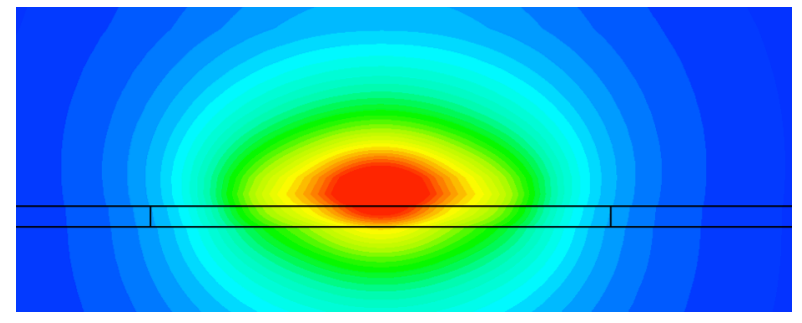

Fig. 3: Cross-sectional view of the field distribution in the center of the gab. The section is normal to the $y$-direction. The horizontal, black lines show the extent of the $\mathrm{SiO}_{2}$ layer. Below the $\mathrm{SiO}_{2}$ is $\mathrm{HR}-\mathrm{Si}$, and above is air. The $\mathrm{SiO}_{2}$ clearly allows most of the field to stay out of the substrate.

The enhancement primarily relies on a time delay between the currents in the antenna - see Fig. 4(a-b). Since the currents in the two loops are both measured positively in the negative $x$-direction, opposite signs means that they are both either charging or discharging the tips. The point where the two reach identical values indicates when the tips reach their maximum charge accumulation. Therefore, the largest, possible field enhancement is obtained if the structure is designed such that the maximum enhancement occurs at the same time as the two currents cross over. In any other case, the tips start to discharge before they have reached their full charge accumulation potential. It is speculated that the entire circuitry can be modelled well by a simple LRC equivalent circuit if given the right way to describe the capacitance of the central gap.
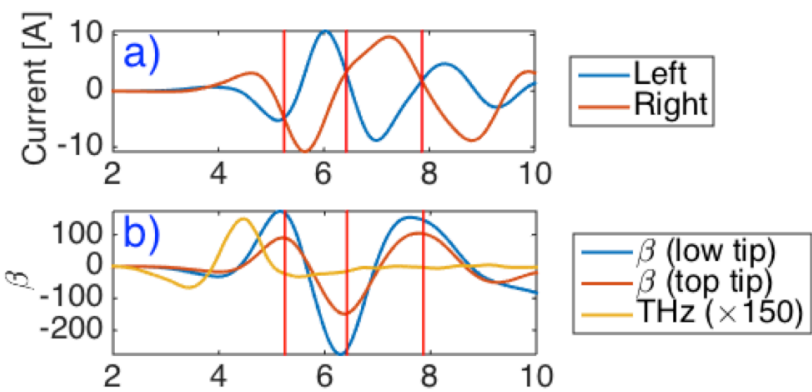

Fig. 4: Time relations between currents, the field enhancement $\beta$ at the tips and the incident $\mathrm{THz}$ pulse (magnified by a factor of 150). The vertical red lines show when the currents in the two loops cross. The cross-over point is closely located to the point where the maximum field enhancement occurs.

In Fig. 5, the gain spectrum can be found for the 3 substrate types $\mathrm{HR}-\mathrm{Si}, \mathrm{SiO}_{2}$ and the $\mathrm{Si} / \mathrm{SiO}_{2}$ hetero-structure. For each substrate, the $\mathrm{THz}$ illumination is either incident on the same side as the metal antennas (brown, orange and green) or on the backside (magenta, blue and red). Illumination from the backside generally gives the highest field enhancement in a gain band up to $0.4 \mathrm{THz}$. The magenta line is for HR-Si, the blue is for the $\mathrm{Si} / \mathrm{SiO}_{2}$ hetero-structure and the red line is for $\mathrm{SiO}_{2}$. For all substrates, the peak resonance of the antenna is determined by the substrate refractive index $n$. A higher $n$ gives a lower resonance. Moreover, the ratio of 1.5 between the antenna loop circumferences leads to visible gain peaks for both antenna resonances as well as the first harmonic for the strongest resonance peak.

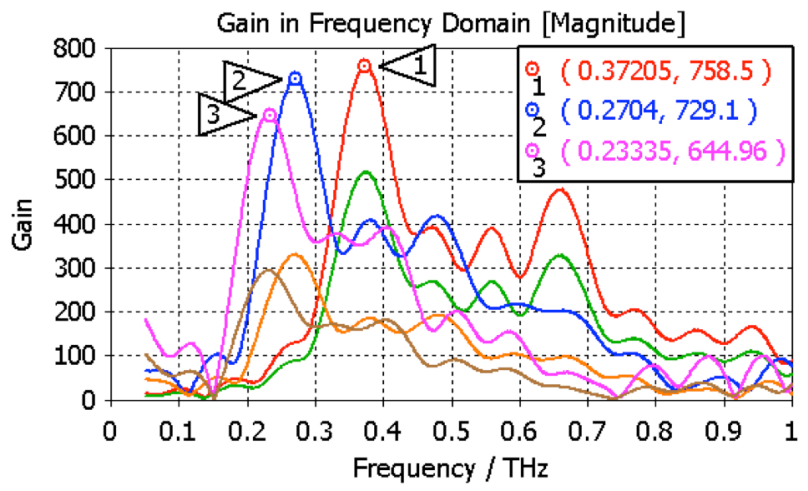

Fig. 5: Gain spectra for $\mathrm{THz}$ radiation incident on the same side of the sample as the metal antennas (front) as well as for the opposite side (back). Colours are: Magenta (HR-Si, back), blue $\left(\mathrm{Si} / \mathrm{SiO}_{2}\right.$, back), red $\left(\mathrm{SiO}_{2}\right.$, back), brown ( $\mathrm{Si}$, front), orange $\left(\mathrm{Si} / \mathrm{SiO}_{2}\right.$, front), green $\left(\mathrm{SiO}_{2}\right.$, front). The two main resonances and the first harmonic for the strongest resonance can be seen in all spectra.

\section{CONCLUSION AND OUTLOOK}

The presented results show that it is possible to create an antenna with high time-domain field enhancement using a multiple-resonant response and a large, $\mu \mathrm{m}$-sized gap. The designed antenna encapsulates the field in a way that could prevent field coupling between antennas when put closely in a large array.

The antenna is placed on a $\mathrm{Si} / \mathrm{SiO}_{2}$ hetero-structure substrate in order to reduce absorption and suppress nonlinear effects close to the antennas. Moreover, it has been shown that the $\mathrm{SiO}_{2}$ layer naturally allows a larger portion of the field to be contained in the air around the antennas rather than in the substrate.

The antenna has the potential to generate a large electron field emission to be used for plasma generation and ultimately to image the incident $\mathrm{THz}$ field.

\section{REFERENCES}

[1]. K. Iwaszczuk, M. Zalkovskij, A. C. Strikwerda and P. U. Jepsen, "Nitrogen plasma formation through terahertz-induced ultrafast electron field emission," Optica, vol. 2, pp. 116-123, 2015.

[2]. Jingdi Zhang et al., "Terahertz radiation-induced sub-cycle field electron emission across a split-gap dipole antenna," Applied Physical Letters, vol. 107, 2015.

[3]. A. C. Strikwerda et al., "Permanently reconfigured metamaterials due to terahertz induced mass transfer of gold," Optics express, vol. 23, pp 11586-99, 2015.

[4] S. Bagiante, F. Enderli, J. Fabianska et al., "Giant Electric Field Enhancement in Split Ring Resonators Featuring Nanometer-Sized Gaps," Scientific Reports, vol. 5, pp. 8051: 1-5, 2015.

[5] A. Tarekegne, K. Iwaszczuk, M. Zalkovskij et al., "Impact ionization in high resistivity silicon induced by an intense terahertz field enhanced by an antenna array," New Journal of Physics, vol. 17, pp. 1-9, 2015. 